Research paper

\title{
Towards mass production of Au nanoparticles supported on montmorillonite microspheres for catalytic reduction of 4-nitrophenol
}

\author{
Fengyan Xiao $^{\mathrm{a}, \mathrm{b}}$, Yawei Qin ${ }^{\mathrm{c}}$, Ning Wang ${ }^{\mathrm{a}, *}$, Dawei Pan ${ }^{\mathrm{a}, *}$ \\ ${ }^{\text {a } C A S ~ K e y ~ L a b o r a t o r y ~ o f ~ C o a s t a l ~ E n v i r o n m e n t a l ~ P r o c e s s e s ~ a n d ~ E c o l o g i c a l ~ R e m e d i a t i o n, ~ R e s e a r c h ~ C e n t e r ~ f o r ~ C o a s t a l ~ E n v i r o n m e n t a l ~ E n g i n e e r i n g ~ a n d ~ T e c h n o l o g y ~ o f ~}$ \\ Shandong Province, Yantai Institute of Coastal Zone Research, Chinese Academy of Sciences, Yantai, Shandong Province 264003, PR China \\ ${ }^{\mathrm{b}}$ Henkel Loctite (China) Co. Ltd, PR China \\ ${ }^{\mathrm{c}}$ CAS Key Laboratory of Engineering Plastics, Institute of Chemistry, Chinese Academy of Sciences, Beijing 100190, PR China
}

\section{A R T I C L E I N F O}

\section{Keywords:}

Au nanoparticles

Montmorillonite

Spherical microspheres

Catalyst

4-nitrophenol

\begin{abstract}
A B S T R A C T
Noble metals supported on substrates have been proven as highly efficient catalysts for the reduction of nitrocompounds. However, their large-scale applications are still limited by the problems of fouling and transport during the mass production process. Herein, we first fabricated a spherical montmorillonite (Mt) substrate via spray drying technique, followed by the deposition of Au NPs through polydopamine chemistry to synthesize spherical Au nanoparticles supported Mt (Au@Mt) microspheres. The Au loading is 14.5 wt $\%$, whereas the specific surface area of the Au@Mt microspheres is $47.3 \mathrm{~m}^{2} \mathrm{~g}^{-1}$, endowing the prepared Au@Mt microspheres with excellent catalytic activity to the reduction of 4-nitrophenol (4-NP) in the presence of $\mathrm{NaBH}_{4} \mathrm{with}$ the optimized apparent reduction rate constant higher than $1.05 \mathrm{~min}^{-1}$. Furthermore, the microspheres can be easily recycled with self-sedimentation without any devices involved and showed excellent stability and recyclability for at least 20 cycles without almost unchanged spherical morphology and catalytic performance. Our straightforward strategy to solve the issue of the mass production process through granulation of amorphous nanomaterial substrate facilitates the practical application of these catalysts in the reduction of nitro-compounds.
\end{abstract}

\section{Introduction}

Recently, noble metal nanoparticles have been the subject of significant scientific and industrial interest due to their unique catalytic properties compared with bulk noble metals (Han et al., 2017; Wang et al., 2017b; Yang et al., 2017). Among them, Au nanoparticles (Au NPs) have been proven as the most efficient catalyst in a series of oxidation and reduction reactions under mild conditions, exhibiting the significant advantages of outstanding catalytic performance, environmental-friendly process, and easy operation (Dhakshinamoorthy et al., 2017; Manzoli et al., 2017; Zugic et al., 2016). Until now, two key issues have been encountered in the attemps of employing Au NPs in pratical catalytic application; indeed, aggregation and recyclablity have been widely considered due to their small size and high surface energy of Au NPs, which heavily affect their catalytic performances and the cost of the catalytic process (Liu et al., 2014). To solve these problems, various materials with the common character of possessing large surface area, easy recovery, and environmental stability, such as polymers (Lucía et al., 2017), carbon materials (Ribeiro et al., 2017), silica materials (Fang et al., 2017), metal oxides (Ruiz and Gianfranco, 2017), and magnetic materials (Wang et al., 2017a), have been used as substrates to support Au NPs. In addition, substrates with the adsorption capacity towards reactants are highly desirable due to the enhanced opportunity for increasing the surface contact between catalyst and reactants (Ma et al., 2015). Through tailoring the surface property of substrates, not only the strong interactions between Au NPs and substrates can be enhanced, but also the adsorption capacity of substrates towards catalytic object can be increased; as a result, the catalytic activity and recyclability of the catalyst can be improved.

One-step generation of Au NPs on the substrates through polydopamine (PDA) chemistry provides a facile and effective strategy to reduce and stabilize Au NPs due to the strong adhesion and the excellent reducing capacity of PDA to reduce Au precursor to Au NPs (Lee et al., 2007). Recently, PDA-functionalized hybrid nanomaterials were applied as novel catalysts to reduce nitro compounds (Arup Kumer et al., 2015; Cao et al., 2015; Luo et al., 2014; Ye et al., 2016; Zeng et al., 2013). For example, Luo et al. fabricated Au NPs supported on PDA-coated graphene catalyst for reduction of 4-nitrophenol (4-NP)

\footnotetext{
* Corresponding authors.

E-mail addresses: nwang@yic.ac.cn (N. Wang), dwpan@yic.ac.cn (D. Pan).
} 
(Luo et al., 2014). Zeng et al. reported a core-shell Fe $\mathrm{O}_{4} @ \mathrm{PDA} @ \mathrm{Au}$ catalyst for the catalytic reduction of nitrobenzene (Zeng et al., 2013). These catalysts demonstrated enhanced adsorption of 4-NP with the help of PDA and high catalytic activity. Moreover, these catalysts could be easily recovered and reused through a magnetic or centrifugal separation.

Despite all the claimed advantages, this promising technique is now limited in the laboratory scale and has yet to be promoted to industrial scale. The challenges, in the authour's opinion, are mainly focused on the mass production and recovery process of the catalyst from the reactive solution without any devices involved. For the production process to be industrially available, the regular morphology and flowability of catalyst are crucial because the amorphous morphology of catalyst not only would result in heavy reactor fouling but would also block the line and, thus, affect the product transport (Yawei et al., 2011). For the recovery process, the relatively large size and sedimentation of catalyst in the reactive solution are significant since the catalyst can be easily recovered from the bottom of treating pond through self-sedimentation without any device involved (Ohta et al., 1980). However, the morphology and sedimentation of the catalyst cannot be found in the literature.

Montmorillonite (Mt) was employed here as the substrate due to its large surface area, excellent adsorption ability, self-sedimentation in the reactive solution, and, most importantly, the fact that it can be granulated (Stunda-Zujeva et al., 2017). We first fabricated a spherical Mt substrate via a spray drying technique, followed by the deposition of $\mathrm{Au}$ NPs through polydopamine chemistry to synthesize spherical Au nanoparticles supported Mt (Au@Mt) microspheres. The model nitrocompound, 4-nitrophenol (4-NP), was selected to evaluate the catalytic performance of $\mathrm{Au} @ \mathrm{Mt}$ microspheres in the present of $\mathrm{NaBH}_{4}$. Furthermore, the recyclability and morphological stability were also investigated in the catalytic process. To the best of our knowledge, this is the first report of fabricated Au@Mt microspheres used as a catalyst for reduction of 4-NP, providing a promising candidate in the large-scale treatment of wastewater containing nitro compounds.

\section{Materials and methods}

\subsection{Materials}

Dopamine hydrochloride (DA) was purchased from Sigma Aldrich (St. Louis, MO, USA). Hydrogen tetrachloroaurate hydrate $\left(\mathrm{HAuCl}_{4} \cdot \mathrm{H}_{2} \mathrm{O}\right)$, 4-nitrophenol (4-NP), and sodium borohydride $\left(\mathrm{NaBH}_{4}\right)$ were obtained from Sinopharm Chemical Reagent Co. Ltd. (China). $1 \mathrm{M}$ Tris-HCl buffer ( $\mathrm{pH}=9.0$ ) was received from Beijing Solarbio Science \& Technology Co., Ltd., China. Montmorillonite (Mt) was kindly supplied by the Fenghong Co. Ltd. (Anji, China). All chemicals and reagents were of analytical grade and used as received. Deionized water was used throughout the work.

\subsection{Granulation of $M t$}

Spherical Mt was fabricated via a spray-drying technique.6.0 $\mathrm{g}$ of Mt was dispersed in $100 \mathrm{~mL}$ of ethanol under 60 -min sonication to obtain the suspension. The suspension was fed into a spray-dryer (YC-015, Shanghai Pilotech Instrument \& Equipment Co. Ltd). The condition of spray-drying was $50 \mathrm{~mL} \mathrm{~min}^{-1}$ feed rate, $110{ }^{\circ} \mathrm{C}$ drying temperature, and $1 \mathrm{~atm}$ compressed-air supply pressure. The yield of spherical Mt was around $45 \%$.

\subsection{Synthesis of spherical Au nanoparticles supported on Mt microspheres}

Spherical Au nanoparticles supported on Mt microspheres (Au@Mt) were synthesized through in situ growth of Au NPs on the surface of PDA-coated Mt Typically, $0.5 \mathrm{~g}$ DA was added into $100 \mathrm{~mL}$ DI water containing $5.0 \mathrm{~g}$ granular Mt and $10 \mathrm{mM}$ Tris- $\mathrm{HCl}$ buffer. The $\mathrm{pH}$ was adjusted to 8.5 using $0.1 \mathrm{M}$ of $\mathrm{NaOH}$ solution. After shaking at ambient conditions for $6 \mathrm{~h}$, the PDA-coated microspheres were filtered from the suspension and further washed with $25 \%$ isopropyl alcohol-water solution for three times. Then, the PDA-coated Mt was dispersed in $50 \mathrm{~mL}$ of an aqueous solution $50 \mathrm{mM}$ of $\mathrm{HAuCl}_{4}$ at ambient conditions under dark for $6 \mathrm{~h}$ using a rotator (MX-RL-Pro, SCILOGEX, USA). After the filter separation, the brown microspheres were dried in a vacuum oven at $60^{\circ} \mathrm{C}$ for $24 \mathrm{~h}$ to obtain the final product Au@Mt.

\subsection{Characterizations}

FTIR spectra were taken in $\mathrm{KBr}$ pressed pellets on a Perkin-Elmer 2000 Fourier transform infrared spectrometer (Connecticut, US). Coldfield-emission scanning electron microscopy (SEM, Jeol S4800, Japan) with an accelerating voltage of $5 \mathrm{kV}$ was used to observe the morphology. Thermogravimetric analysis (TGA) measurements were performed on Pyris TGA-7A (Perkin-Elmer, US) in the air with a purge rate of $50 \mathrm{~mL} \mathrm{~min}^{-1}$. X-ray diffraction (XRD) patterns were recorded on a Bruker D8-Advantage powder diffractometer (Karlsruhe, Germany) using $\mathrm{Cu} \mathrm{Ka}$ radiation $(40 \mathrm{kV}, 110 \mathrm{~mA})$. X-ray photoelectron spectroscopy (XPS, Thermo EscaLab 250Xi, Thermo Fisher Scientific, US) was used to examine the surface composition with monochromatic $\mathrm{Al} \mathrm{K \alpha}$ excitation ( $\mathrm{h} \nu=1486.6 \mathrm{eV}$ ) on a spot size of $500 \mu \mathrm{m}$. Nitrogen adsorption experiments on samples were carried out at $77 \mathrm{~K}$ with an ASAP2020 (Micromeritics, US). BET surface area, pore size, and pore volume were evaluated via the conventional analysis of the nitrogen isotherms. The UV absorption spectra of the samples were obtained on a UV-vis spectrometer (PERSEE UT 1810, Shanghai, China).

\subsection{Adsorption experiment}

$20 \mathrm{mg}$ of each microsphere were added to $100 \mathrm{~mL}$ of $100 \mathrm{mg} \mathrm{L}^{-1} 4$ $\mathrm{NP}$ aqueous solution under constant stirring at room temperature. UV-vis absorption spectra of 4-NP at different intervals were recorded to monitor the adsorption process. The removal percentage of 4-NP is calculated following the Eq. (1) (Wang et al., 2017c):

$\mathrm{R}_{4-\mathrm{NP}}=\left(\frac{\mathrm{C}_{0}-\mathrm{C}_{\mathrm{t}}}{\mathrm{C}_{0}}\right) \times 100 \%$

Where $\mathrm{R}_{4-\mathrm{NP}}$ is the removal percentage of 4-NP, the $\mathrm{C}_{\mathrm{o}}\left(\mathrm{mg} \mathrm{L}^{-1}\right)$ and $\mathrm{C}_{\mathrm{t}}\left(\mathrm{mg} \mathrm{L}^{-1}\right)$ are the initial dye concentration and the equilibrium concentration, respectively.

\subsection{Catalytic reduction of 4-NP}

The catalytic reduction of 4-NP in the presence of $\mathrm{NaBH}_{4}$ was carried out to assess the catalytic activity and reusability of the Au@Mt microsphere. $1 \mathrm{~mL} 4-\mathrm{NP}\left(250 \mathrm{mg} \mathrm{L}^{-1}\right)$ and $2 \mathrm{~mL} \mathrm{NaBH}_{4}(0.1 \mathrm{M})$ aqueous solutions were mixed with $7 \mathrm{~mL}$ deionized water, followed by the addition of 2 mg Au@Mt microspheres. The yellow-green color of 4-NP vanished as the reaction proceeded; the process was monitored at $400 \mathrm{~nm}$ using a UV-vis spectrometer. After the whole reduction process was completed, the Au@Mt microsphere was separated from the mixture with self-sedimentation and, then, reused in the next cycle. The concentrations of $\mathrm{Au} @ \mathrm{Mt}$, 4-NP, and $\mathrm{NaBH}_{4}$ were investigated to influence their apparent reduction rate constants.

\section{Results and discussion}

\subsection{Synthesis and characterization of the Au@Mt microspheres}

Spray-drying is a common technique to fabricate granulation in industry (Loghman-Estarki et al., 2013). PDA has exhibited strong adhesion towards substrates and excellent capacity to reduce Au precursor to Au NPs (Lee et al., 2007). Therefore, a simple approach was applied 

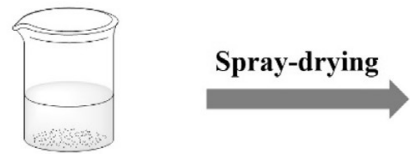

Mt/Ethanol Suspension

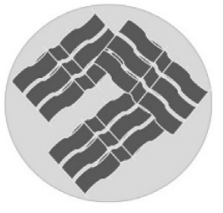

Spherical Mt

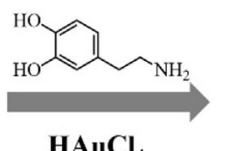

$\mathrm{HAuCl}_{4}$

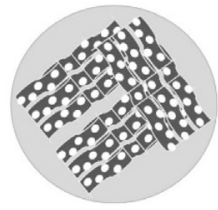

SphericalAu@Mt
Scheme 1. Synthesis and characterization of the $\mathrm{Au} @ \mathrm{Mt}$ microspheres, including the first step of spray drying and the second step of PDA coating and dispersion into the $\mathrm{HAuCl}_{4}$ solution. to fabricate spherical Au NPs supported Mt microspheres via PDA chemistry, as shown in Scheme 1. Mt was firstly mixed with ethanol and, thus, granulated via a spray-drying technique. Then, the spherical Mt was added to dopamine solution at $\mathrm{pH}$ 8.5. After 8-h polymerization under shaker, the PDA-coated spherical Mt was washed thoroughly and collected through filtration. After that, the PDA-coated spherical Mt was then dispersed in $\mathrm{HAuCl}_{4}$ solution under rotator in the dark for $12 \mathrm{~h}$. The final product was collected and dried to obtain Au@Mt.

Through granulation process, not only the regularity of the product can be improved and the fouling can be decreased, but also the flowability of the product can be enhanced, thereby the transport in the product-line can be facile achieved (Stunda-Zujeva et al., 2017). Therefore, the morphology of product is an essential step to successfully implement the large-scale production. SEM was employed to observe the morphologies of Mt through granulation process, PDA@Mt, and Au@Mt microspheres. As shown in Fig. 1, the Mt through spray-drying exhibits a spherical particle morphology with an average diameter of $20 \mu \mathrm{m}$. The XRD pattern (inset in Fig. 1a) illustrates the (001) diffraction peak at $2 \theta=7.23^{\circ}$, corresponding to a basal distance of $0.96 \mathrm{~nm}$, in agreement with the result of pristine Mt in our previous work (Wang et al., 2017c). These results indicate an unchanged lamellar structure of Mt after the granulation process. The nitrogen adsorption-desorption isotherms of the spherical Mt obtained at $77 \mathrm{~K}$ are shown in Fig. 1d. The nitrogen adsorption and desorption isotherms of spherical Mt exhibit a regular pore structure of a mesoporous material, giving a BET specific surface area of the spherical Mt at $60.6 \mathrm{~m}^{2} \mathrm{~g}^{-1}$ and a total pore volume of $0.38 \mathrm{~cm}^{-3} / \mathrm{g}$. The specific surface area and total pore volume of spherical Mt in our experiment are much higher than that of Mt as
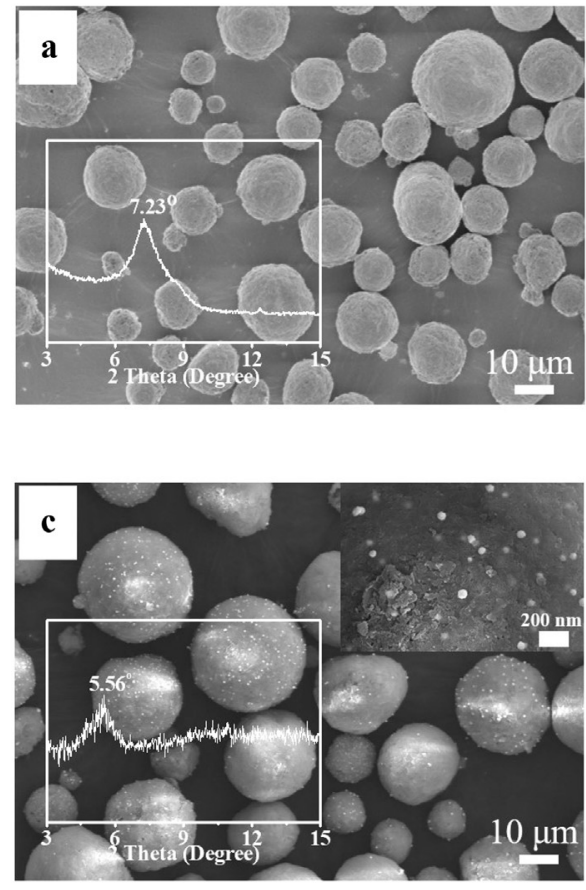

received $\left(8.3 \mathrm{~m}^{2} \mathrm{~g}^{-1}\right.$, Fig. S1) and as reported in the literature $\left(9.0 \mathrm{~m}^{2} \mathrm{~g}^{-1}\right)$ (Gil et al., 2011), suggesting the efficient pore-making of a spray-drying process. As the spherical Mt was well prepared, the fabrication of Au NPs supported Mt microspheres easily fixed the Au NPs on the spherical Mt for catalytic reduction of 4-NP. As shown in Fig. 1c, the morphology of spherical Mt is unchanged after the supported process. Small bright particles with a average diameter of $40 \mathrm{~nm}$ can be observed on the surface of spherical Mt and are associated to the Au NPs peak via XRD shown in Fig. S2 (Zeng et al., 2013). The XRD patterns (inset in Fig. 1b) show the (001) diffraction peak at $2 \theta=5.56^{\circ}$, corresponding to a basal distance of $1.56 \mathrm{~nm}$ after coating with PDA, suggesting that the coating on each side of Mt layer is $0.3 \mathrm{~nm}$. However, after generating Au NPs, the diffraction peak position of (001) of Mt did not change. It may be only illustrated that most of Au NPs existed in the outside surface of aggregated silicate layers (inset in Fig. 1c). Compared with the spherical Mt, the specific surface area and total pore volume of $\mathrm{Au} @ \mathrm{Mt}$ microspheres slightly decrease to $47.3 \mathrm{~m}^{2} \mathrm{~g}^{-1}$ and $0.31 \mathrm{~cm}^{-3}$ / g. The loading of Au NPs on Au @Mt microspheres is calculated to be $14.5 \mathrm{wt} \%$ from the weight loss differences of Mt, PDA-coated spherical Mt (PDA@Mt), and Au@Mt microspheres as shown in Fig. S3. Furthermore, the details of calculation for Au loading amount by TGA results are shown in the supplementary data. The Au loading in our experiment is much higher than that on $\mathrm{Fe}_{3} \mathrm{O}_{4}$ substrate $(4.3 \mathrm{wt} \%)$ (Zeng et al., 2013), suggesting the efficient Au NPs deposition on spherical Mt The high Au loading and large specific surface area of Au@ Mt microspheres indicate good adsorption and catalytic reduction capability towards organic dyes.
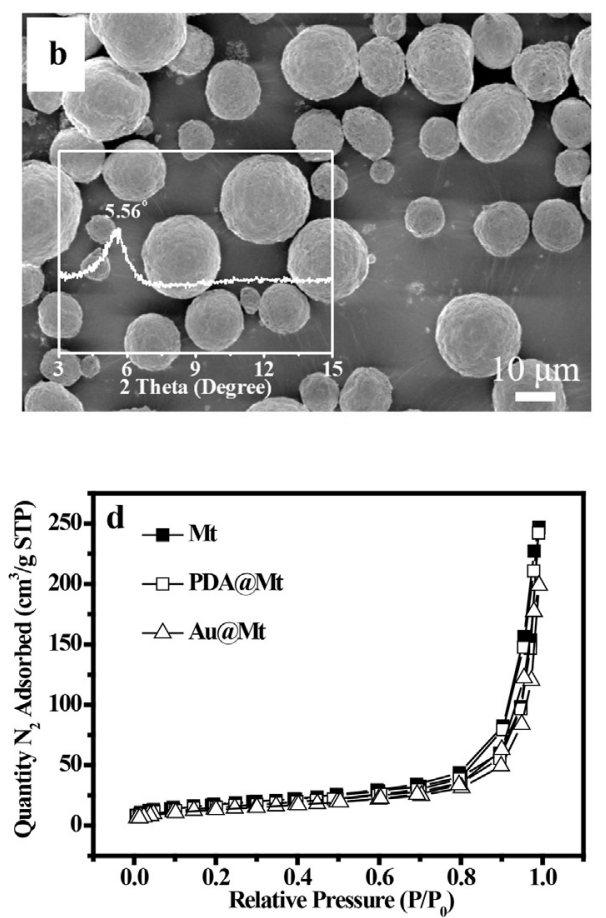

Fig. 1. SEM images of the spherical Mt (a), PDA@Mt (b), Au@Mt microspheres (c), and $\mathrm{N}_{2}$-sorption profiles of the spherical Mt, PDA-coated spherical Mt, and Au@ Mt microspheres (d), with inset XRD patterns, demonstrating the ordering state of silicate layers; the nanoparticles size of Au NPs. 


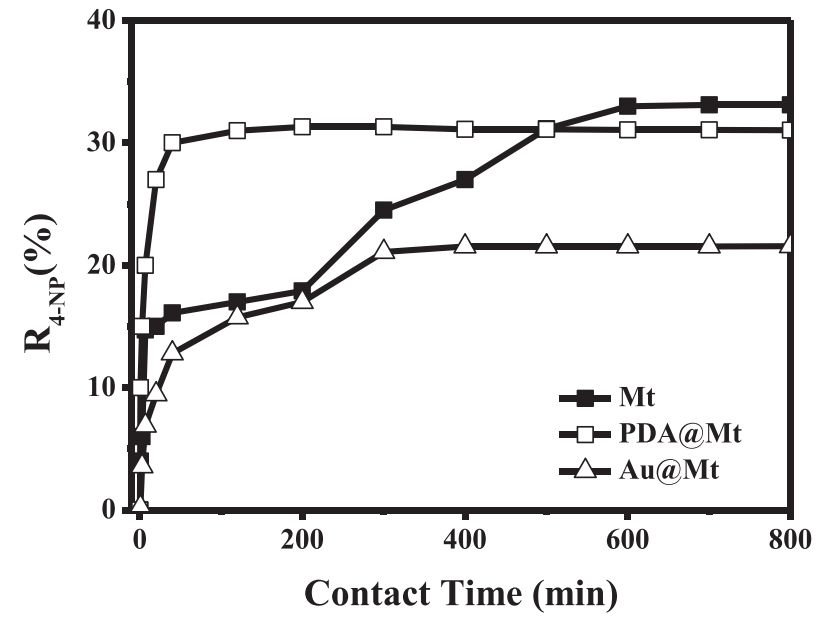

Fig. 2. The adsorption kinetics of 4-NP on the spherical Mt, PDA-coated spherical Mt, and Au@Mt microspheres.

\subsection{Adsorption of Au@Mt microspheres}

The adsorption capability towards organic dyes can remarkably improve the catalytic activity of noble metal catalysts due to the enhanced contact between the catalyst and organic dyes (Zhang et al., 2014). 4-NP was chosen as the model dye. The band of 4-NP is known to appear at $400 \mathrm{~nm}$; therefore, the change of the 4-NP peak during the adsorption process is feasibly monitored via time-dependent UV-Vis absorption spectra (Zhang et al., 2014). All three kinds of samples, including the spherical Mt, PDA-coated spherical Mt, and Au@Mt microspheres, exhibit a similar adsorption behavior with three stages. After an initial fast adsorption process, the adsorption gradually increases and finally reaches the equilibrium. Although the three samples present a similar fast adsorption process, the gradual adsorption stages are different due to the difference of the intra-particle diffusion-rate controlling step. As shown in Fig. 2, compared with the spherical Mt, the intra-particle diffusion stage is shortened for PDA@Mt due to the adsorb 4-NP by $\pi-\pi$ stacking interaction between 4-NP and PDA. The deposition of Au NPs on PDA@Mt significantly prolongs the intraparticle diffusion rate. At last, all three samples reach the equilibrium stage. The max adsorption of PDA@Mt is similar with that of spherical Mt, whereas Au@Mt microspheres show a relative lower adsorption capability because Au NPs occupy the surface of PDA@Mt and, thus, reduce the adsorption capacity of 4-NP.

\subsection{Catalytic properties of Au@Mt microspheres}

The catalytic capacity of Au@Mt microspheres was evaluated through the color variation of 4-NP undergoing reduction in the presence of $\mathrm{NaBH}_{4}$. At the beginning of 4-NP reduction, the peak at $400 \mathrm{~nm}$ decreases its intensity. Accordingly, a new peak at 300 appears due to the formation of 4-aminophenol (4-AP). Therefore, the catalytic reduction process of 4-NP was monitored by its time-dependent absorbance change using UV-vis spectroscopy. As shown in Fig. 3a, with the prolonged reduction of 4-NP progressing, the absorbance peak of 4-NP at $400 \mathrm{~nm}$ rapidly decreases to zero in $7 \mathrm{~min}$, while a new peak at $300 \mathrm{~nm}$ appears and gradually increases. Accordingly, the green-yellow color of 4-NP solution concomitantly disappears. The catalytic time of $\mathrm{Au} @ \mathrm{Mt}$ microspheres in the reduction of 4-NP is much less than the Au NPs reported in the literature, revealing the high catalytic efficiency of the prepared Au@Mt microspheres. The catalytic rate is highly related to the surface area of Au NPs supplied for the electron relay from $\mathrm{NaBH}_{4}$ to 4-NP. Therefore, the reaction kinetics of the reduction of the 4-NP under different catalytic conditions are calculated by monitoring their time-dependent UV-Vis absorption spectra. From these spectra, the pseudo-first-order reaction kinetics are used to calculate the reaction rate constant. From the linear relation of $\ln \left(\mathrm{C}_{t} / \mathrm{C}_{0}\right)$ with the reaction time, the apparent reduction rate constant $(\mathrm{K})$ of $4-\mathrm{NP}$ is obtained. Fig. $3 \mathrm{~b}$ shows that $\mathrm{K}$ increases linearly from $0.08 \mathrm{~min}^{-1}$ to $0.95 \mathrm{~min}^{-1}$ with the Au@Mt microspheres concentration increasing from $200 \mathrm{mg} \mathrm{L}^{-1}$ to $1000 \mathrm{mg} \mathrm{L}^{-1}$, when the concentrations of $\mathrm{NaBH}_{4}$ and 4$\mathrm{NP}$ are fixed at $0.02 \mathrm{M}$ and $250 \mathrm{mg} \mathrm{L}^{-1}$, respectively. The increased $\mathrm{K}$ is mainly due to the increased total available surface area of Au NPs.

$\mathrm{K}$ is also determined by the surface coverage degree by each reactant, according to the following Eq. (2) (Wunder et al., 2011):

$\mathrm{K}=\mathrm{kS}_{4-\mathrm{NP}} \theta_{\mathrm{BH}_{4}^{-}}$

Where $k$ is the molar rate constant per square meter of the catalyst, $S$ is the total available surface area of Au NPs, $\theta_{4-N P}$ and $\theta_{B H_{4}}{ }^{-}$are the surface coverage degree by 4-NP and $\mathrm{BH}_{4}{ }^{-}$. The competition between 4$\mathrm{NP}$ and $\mathrm{BH}_{4}{ }^{-}$on the surface of Au NPs can affect $\mathrm{K}$. The excess of 4-NP results in the decrease of $\mathrm{K}$, while the excess of $\mathrm{NaBH}_{4}$ leads to the increase of $\mathrm{K}$ due to the limited surface area of Au NPs. K decreases linearly from $1.05 \mathrm{~min}^{-1}$ to $0.08 \mathrm{~min}^{-1}$ with 4-NP concentration increasing from $100 \mathrm{mg} \mathrm{L}^{-1}$ to $350 \mathrm{mg} \mathrm{L}^{-1}$ (Fig. 3c). According to Eq. (2), $\mathrm{K}$ decreases with the increase of 4-NP and is mainly due to the increasing $\theta_{4-N P}$ and the decreasing $\theta_{B_{4}}{ }^{-}$that reduce the rate of electron relay from $\mathrm{NaBH}_{4}$ to 4-NP. On the contrary, as shown in Fig. 3d, the increasing $\theta_{\mathrm{BH}_{4}}{ }^{-}$and the decreasing $\theta_{4-N P}$ improve the rate of electron relay from $\mathrm{NaBH}_{4}$ to 4-NP. Above all, these results indicate that $\mathrm{K}$ of the so-prepared Au@Mt can be optimized by tailoring the composition of $\mathrm{Au} \mathrm{NPs}$, 4-NP, and $\mathrm{NaBH}_{4}$. Interestingly, the $\mathrm{K}\left(1.05 \mathrm{~min}^{-1}\right)$ in our fabricatedAu@Mt microspheres is much higher than the value on other substrates $\left(0.23 \mathrm{~min}^{-1}\right.$ (Luo et al., 2014) and $0.63 \mathrm{~min}^{-1}$ (Zeng et al., 2013) for graphene oxide and $\mathrm{Fe}_{3} \mathrm{O}_{4}$, respectively) reported in the literatures. Furthermore, the $\mathrm{K}$ over the Au weight of catalyst was calculated as $60 \mathrm{~s}^{-1} / \mathrm{g} \mathrm{Au}$, which is similar with that of reported $\left(90 \mathrm{~s}^{-1} / \mathrm{g}\right.$ $\mathrm{Au}$, (Luo et al., 2014)). In addition, the turnover frequency (TOF) of catalyst was calculated as $0.22 \mathrm{~min}^{-1}$, according to the moles of organic dye catalytic reduced by per mole of Au under the consumed time, which is higher than that of previous reported $\left(0.10 \mathrm{~min}^{-1}\right.$, (Luo et al., 2014)). The superior high catalytic activity of Au@Mt microspheres can be attributed to two factors: (1) the well-dispersed and highly stable Au NPs on the chemically stable Mt support, and (2) the large surface area and strong adsorption capacity of granular $\mathrm{Mt}$ (Fig. 4a).

\subsection{Recyclability examination}

Recyclability is one of the most critical properties of a catalyst to maintain their efficient catalytic performances for practical applications. The successive 20 catalytic cycles of Au@Mt microspheres were carried out to determine its recyclability. Fig. $4 \mathrm{~b}$ shows that the catalytic performances of Au@Mt microspheres are almost unchanged even after 20 cycles. Furthermore, the K slightly decreases $10 \%$ from the 1st to the 20th cycle, as displayed in Fig. 4c. In addition, the SEM image of Au@Mt microspheres after 20 cycles exhibits well-maintained structure and morphology, which provides an undeniable evidence for the excellent stability of the prepared catalyst (Fig. 4d). The outstanding recyclability and stability of our prepared Au@Mt microspheres mainly depend on the stability of Mt and PDA-coating because Mt and PDA are very stable in the aqueous solution (Xie et al., 2014). Therefore, all these advantages of Au@Mt microspheres indicate a great potential in large-scale application.

\section{Conclusions}

By fabricating spherical nanomaterials through spray drying technology, the issues of fouling and transport during the mass production were successfully solved. We synthesized and characterized Au@Mt microspheres, by systematically investigating their stability, catalytic 
a
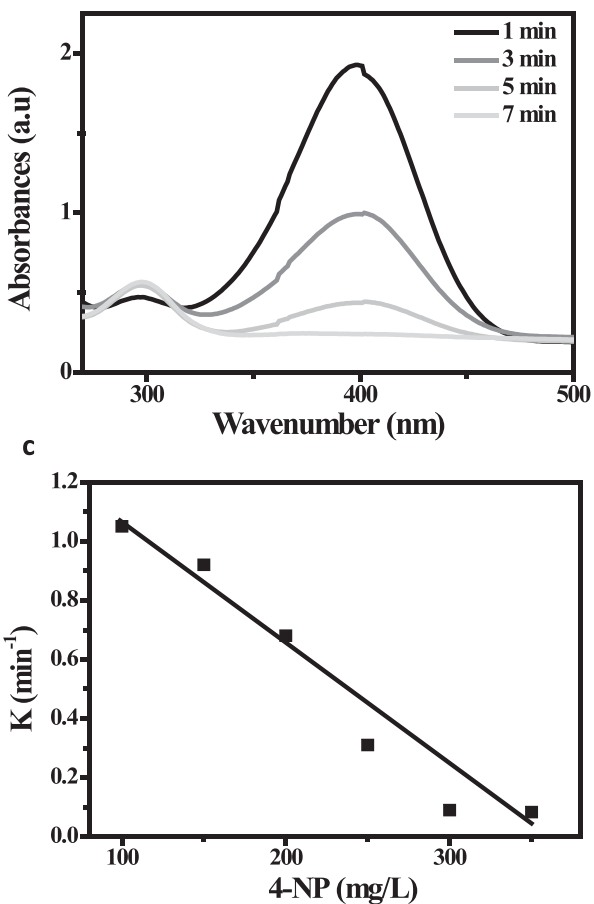

b
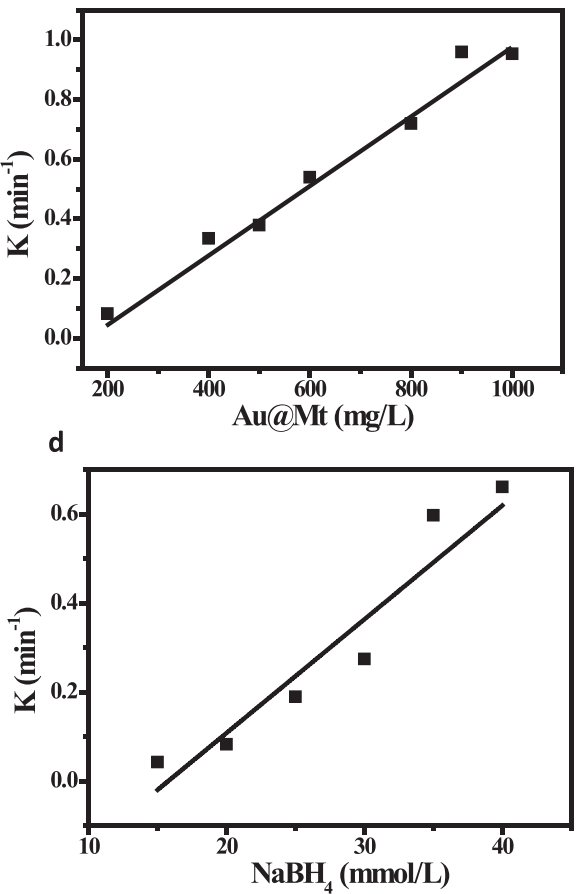

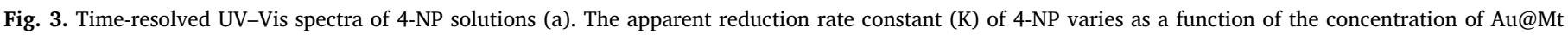
microspheres (b), 4-NP (c), and $\mathrm{NaBH}_{4}$ (d) concentration, respectively.

performance, and recyclability. The prepared Au@Mt microspheres presented a high catalytic performance towards 4-NP in the presence of $\mathrm{NaBH}_{4}$ due to well-dispersed Au NPs on the spherical Mt substrate and the strong 4-NP adsorption capacity of spherical Mt Furthermore, the microspheres can be easily recycled with self-sedimentation without any devices involved and showed excellent stability and recyclability for at least 20 cycles, with almost unchanged spherical morphology and catalytic performance. Our straightforward approach to solving the problem of the production process through granulation of amorphous nanomaterials may pave a new way to massively produce high-performance catalysts for practical applications. a

\section{$\mathrm{NaBH}_{4}$}

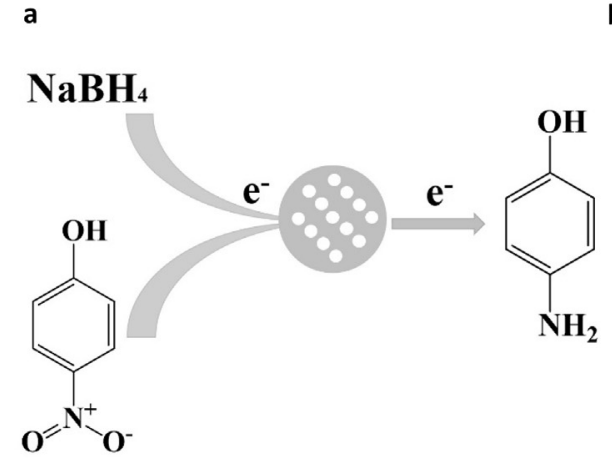

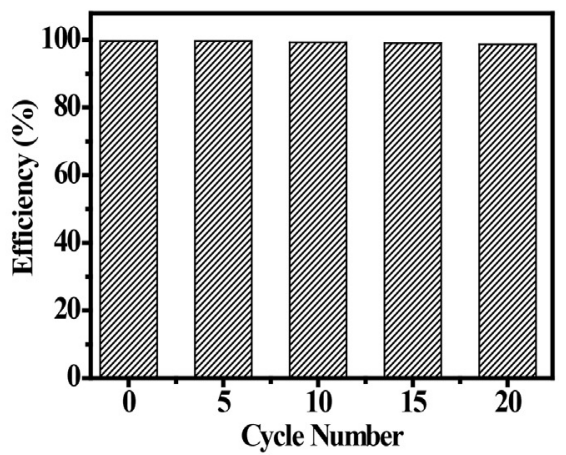

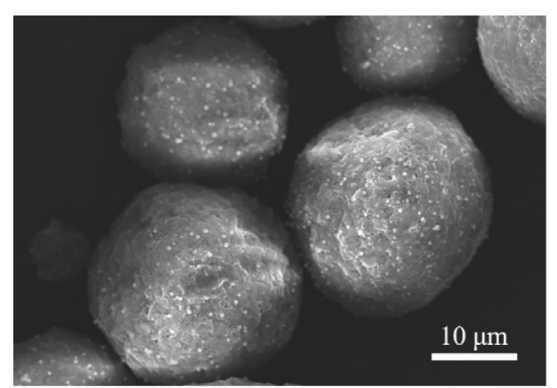

c

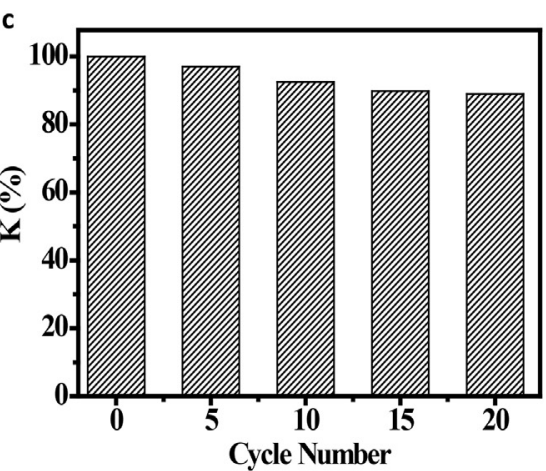

d

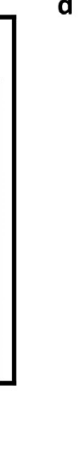

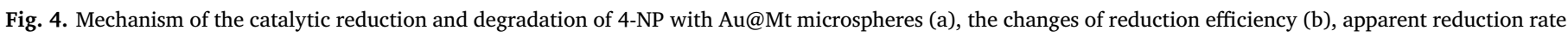

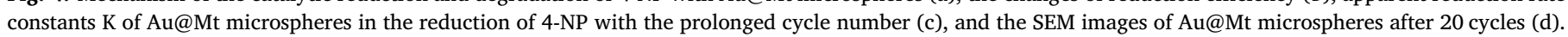




\section{Author contributions}

All authors have given approval to the final version of the manuscript. Fengyan Xiao and Yawei Qin contributed equally to this work.

\section{Acknowledgments}

The authors gratefully acknowledge the funding support from National Natural Science Foundation of China (No. 51703110), Key Research and Development Program of Shandong Province (No. 2016ZDJS04B03), Key Science and Technology Program of Yantai City (No. 2018ZHGY075), and the MogoEdit for their language editing service.

\section{Appendix A. Supplementary data}

Supplementary data to this article can be found online at https:// doi.org/10.1016/j.clay.2018.09.016.

\section{References}

Arup Kumer, R., Sung Young, P., Insik, I., 2015. Mussel-inspired synthesis of boron nitride nanosheet-supported gold nanoparticles and their application for catalytic reduction of 4-nitrophenol. Nanotechnology 26, 105601.

Cao, J., Mei, S., Jia, H., Ott, A., Ballauff, M., Lu, Y., 2015. In Situ synthesis of catalytic active Au nanoparticles onto gibbsite-polydopamine core-shell nanoplates. Langmuir 31, 9483-9491.

Dhakshinamoorthy, A., Asiri, A.M., Garcia, H., 2017. Metal organic frameworks as versatile hosts of Au nanoparticles in heterogeneous catalysis. ACS Catal. 7, 2896-2919.

Fang, J., Zhang, Y., Zhou, Y., Zhao, S., Zhang, C., Zhang, H., Sheng, X., Wang, K., 2017. Fabrication of ellipsoidal silica yolk-shell magnetic structures with extremely stable Au nanoparticles as highly reactive and Recoverable catalysts. Langmuir 33, $2698-2708$.

Gil, A., Assis, F.C.C., Albeniz, S., Korili, S.A., 2011. Removal of dyes from wastewaters by adsorption on pillared clays. Chem. Eng. J. 168, 1032-1040.

Han, J., Wang, M., Hu, Y., Zhou, C., Guo, R., 2017. Conducting polymer-noble metal nanoparticle hybrids: Synthesis mechanism application. Prog. Polym. Sci. 70, 52-91.

Lee, H., Dellatore, S.M., Miller, W.M., Messersmith, P.B., 2007. Mussel-inspired surface chemistry for multifunctional coatings. Science 318, 426-430.

Liu, Y., Ai, K., Lu, L., 2014. Polydopamine and its derivative materials: synthesis and promising applications in energy, environmental, and biomedical fields. Chem. Rev. 114, 5057-5115.

Loghman-Estarki, M.R., Edris, H., Razavi, R.S., Jamali, H., Ghasemi, R., Pourbafrany, M., Erfanmanesh, M., Ramezani, M., 2013. Spray drying of nanometric SYSZ powders to obtain plasma sprayable nanostructured granules. Ceram. Int. 39, 9447-9457.

Lucía, M.T., Vicente, E.P., Ocampo, A.L., Eduardo, R.D.S.M., Daniel, G., Josefina, D.G. 2017. Simultaneous AuIII extraction and in situ formation of polymeric membranesupported Au nanoparticles: a sustainable process with application in catalysis. ChemSusChem 10, 1482-1493.

Luo, J., Zhang, N., Liu, R., Liu, X., 2014. In situ green synthesis of Au nanoparticles onto polydopamine-functionalized graphene for catalytic reduction of nitrophenol. RSC Adv. 4, 64816-64824.

Ma, A., Xie, Y., Xu, J., Zeng, H., Xu, H., 2015. The significant impact of polydopamine on the catalytic performance of the carried Au nanoparticles. Chem. Commun. 51, 1469-1471.

Manzoli, M., Vindigni, F., Tabakova, T., Lamberti, C., Dimitrov, D., Ivanov, K., Agostini, G., 2017. Structure-reactivity relationship in $\mathrm{Co} 3 \mathrm{O} 4$ promoted $\mathrm{Au} / \mathrm{CeO}_{2}$ catalysts for the $\mathrm{CH}_{3} \mathrm{OH}$ oxidation reaction revealed by in situ FTIR and operando EXAFS studies. J. Mater. Chem. A 5, 2083-2094.

Ohta, H., Goto, S., Teshima, H., 1980. Liquid-phase oxidation of phenol in a rotating catalytic basket reactor. Indonesia 19, 180-185.

Ribeiro, A.P.C., Martins, L.M.D.R.S., Carabineiro, S.A.C., Figueiredo, J.L., Pombeiro, A.J.L., 2017. Gold nanoparticles deposited on surface modified carbon materials as reusable catalysts for hydrocarboxylation of cyclohexane. Appl. Catal. A-Gen. 547, $124-131$.

Ruiz, P.A., Gianfranco, P., 2017. CO Oxidation on Au nanoparticles supported on $\mathrm{ZrO} 2$ role of metal/oxide interface and oxide reducibility. ChemCatChem 9, 1119-1127.

Stunda-Zujeva, A., Irbe, Z., Berzina-Cimdina, L., 2017. Controlling the morphology of ceramic and composite powders obtained via spray drying-a review. Ceram. Int. 43 , $11543-11551$.

Wang, D., Duan, H., Lu, J., Lu, C., 2017a. Fabrication of thermo-responsive polymer functionalized reduced graphene oxide@ $\mathrm{Fe}_{3} \mathrm{O}_{4} @ \mathrm{Au}$ magnetic nanocomposites for enhanced catalytic applications. J. Mater. Chem. A 5, 5088-5097.

Wang, L., Zeng, Z., Ma, C., Liu, Y., Giroux, M., Chi, M., Jin, J., Greeley, J., Wang, C., 2017b. Plating precious metals on nonprecious metal nanoparticles for sustainable electrocatalysts. Nano Lett. 17, 3391-3395.

Wang, N., Hu, Y., Zhang, Z., 2017c. Sustainable catalytic properties of silver nanoparticles supported montmorillonite for highly efficient recyclable reduction of methylene blue. Appl. Clay Sci. 150, 47-55.

Wunder, S., Lu, Y., Albrecht, M., Ballauff, M., 2011. Catalytic activity of faceted gold nanoparticles studied by a model reaction: evidence for substrate-induced surface restructuring. ACS Catal. 1, 908-916.

Xie, Y., Yan, B., Xu, H., Chen, J., Liu, Q., Deng, Y., Zeng, H., 2014. Highly regenerable mussel-inspired Fe304@ polydopamine-Ag core-shell microspheres as catalyst and adsorbent for methylene blue removal. ACS Appl. Mater. Interfaces 6, 8845-8852.

Yang, Q., Xu, Q., Jiang, H.-L., 2017. Metal-organic frameworks meet metal nanoparticles: synergistic effect for enhanced catalysis. Chem. Soc. Rev. 46, 4774-4808.

Yawei, Q., Ning, W., Yong, Z., Yingiuan, H., Hui, N., Jin-Yong, D., 2011. Fabrication of nanofillers into a granular "nanosupport" for ziegler-natta catalysts: towards scalable in situ preparation of polyolefin nanocomposites. Macromol. Rapid Commun. 32, 1052-1059.

Ye, W., Yu, J., Zhou, Y., Gao, D., Wang, D., Wang, C., Xue, D., 2016. Green synthesis of $\mathrm{Pt}-\mathrm{Au}$ dendrimer-like nanoparticles supported on polydopamine-functionalized graphene and their high performance toward 4- nitrophenol reduction. Appl. Catal., B $181,371-378$.

Zeng, T., Niu, H.-y., Ma, Y.-r., Li, W.-h., Cai, Y.-q., 2013. In situ growth of gold nanoparticles onto polydopamine-encapsulated magnetic microspheres for catalytic reduction of nitrobenzene. Appl. Catal., B 134, 26-33.

Zhang, Y., Zhu, P., Chen, L., Li, G., Zhou, F., Lu, D.D., Sun, R., Zhou, F., Wong, C.-p., 2014. Hierarchical architectures of monodisperse porous Cu microspheres: synthesis, growth mechanism, high-efficiency and recyclable catalytic performance. J. Mater. Chem. A 2, 11966-11973.

Zugic, B., Wang, L., Heine, C., Zakharov, D.N., Lechner, B.A.J., Stach, E.A., Biener, J., Salmeron, M., Madix, R.J., Friend, C.M., 2016. Dynamic restructuring drives catalytic activity on nanoporous gold-silver alloy catalysts. Nat. Mater. 16, 558. 